\title{
Surface Morphology and Stability Analysis of Ceria-based Nanoparticles for its Utilization as a Lubricant Additive
}

\author{
J. Philip ${ }^{1 *}$, C. Koshy ${ }^{2}$ \\ ${ }^{1}$ Saintgits College of Engineering, Kottayam, India \\ ${ }^{2}$ National Institute of Technology, Calicut, India \\ \{philip.jibin07@gmail.com\}
}

\begin{abstract}
The lubricants added with nanoparticle additives have achieved a predominant position in the field of research on development of sustainable lubricating oils. The efficient physical and chemical properties of metal oxide nanoparticles as lubricant additives have widely extended its purview. The surface morphology and stability analysis of nano-sized Ceria $\left(\mathrm{CeO}_{2}\right)$ and Ceria-Zirconia $(\mathrm{Ce}-\mathrm{Zr})$ hybrid nanoparticles are carried out using High Resolution Transmission Electron Microscopy (HR-TEM), Fourier Transform Infrared Spectroscopy (FT-IR), X-Ray Diffraction (XRD) and Zeta Potential (ZP) analyses techniques. The ceria-based nanoparticles are synthesized through the bottom-up approach of chemical processing named Precipitation Method (PM). The better control on particle size of PM resulted in refined and spherically shaped nanoparticles. Further, to improve the dispersion stability of the prepared nanoparticles in the lubricant, a suitable surfactant namely Polysorbate 20 (Tween 20) was used. The characterization results proved that the ceria-based nanoparticles can play an important role in improving the lubricating properties of the base-oil, provided that proper measures are taken to compensate for its minimal undesirable effects.
\end{abstract}

Keywords:Lubricant, Nanoparticles, Morphology, Precipitation Method, Surfactant

\section{Introduction}

Nickel based alloys finds wide applications in different parts of gas turbines of aircraft, steam turbine power plants, reciprocating engines, tool \& die industry, medical field, heat treatment equipments, nuclear power plants, The establishment of the concept of nanotechnology and its widespread acceptance has led to the study and development of materials at atomic scales. The positive change in physical and chemical properties of elements at nano/micrometre size has given a constructive leap in various fields of research such as chemistry, nano technology, material science and Engineering. The new challenges and demands in advanced technologies have forced the investigators to think and derive solutions to reduce or prevent the energy and material losses in various systems.

Diminishing fossil fuels and crude oil reserves along with its depleting effects on the environment has alarmed the need for establishment of new bio-degradable and sustainable energy sources. The central reason behind energy loss in mechanical systems viz., automobiles, industrial machineries etc. is recognized to be the phenomenon of friction. The most effective method derived so far for the reduction of friction and wear is the technique of lubrication. Recently, the addition of nanoparticles in lubricants and fuels to enhance its rheological, thermal and tribological properties is obtaining widespread attention due to its enhanced positive effects. The basic principle of nanolubrication is an inevitable factor needed for the field to advance and certain modifications done at accurate levels can impart effective surface protection [1]. Currently, many researches are being carried out in the field of nanolubrication to explore the possibilities in development of sustainable, ecofriendly and bio-degradable lubricants. Many investigators have identified that the addition of nanoparticles can magnify the required properties of lubricating oils, if properly mixed in definite ratios [2-6].

\subsection{Nanoparticle Additives: State of Art}

The synthesis of nanoparticles is achieved by two processes; viz., physical processes and chemical processes.

B. Iyer, S. Nalbalwar and R. Pawade (Eds.)

ICCASP/ICMMD-2016. Advances in Intelligent Systems Research.

Vol. 137, Pp. 316-323.

(C) 2017. The authors - Published by Atlantis Press

This is an open access article under the CC BY-NC license (http://creativecommons.org/licens)es/by-nc/4.0/).

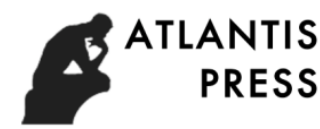


The physical processes that are widely used include high energy ball milling, inert gas condensation, wire explosion, ion sputtering, arc discharge, laser ablation, etc. However, the need for large energy sources and ineffective control on particle size make the physical processes non-economical. In chemical methods, nanoparticles are developed starting from atoms. They include chemical reduction, sol-gel method, precipitation method, solvo-thermal synthesis, photo chemical synthesis, electro-chemical synthesis, etc. [7-11]. A wide range of nanoparticles such as $\mathrm{MoS}_{2}, \mathrm{CuO}, \mathrm{TiO}_{2}, \mathrm{ZnO}_{2}$, etc. have been tested as lubricant additives by many researchers in the past. The major reasons for the utilization of nanoparticles as lubricant additives are due to its shape, less chemical reactivity, non-volatility etc. Further, the size, shape, concentration and dispersion stability of the nanoparticles are the major factors which determine the friction reduction and anti-wear properties of the nanolubricants [12].

In this work, the nano-sized Ceria $\left(\mathrm{CeO}_{2}\right)$ and Ceria-Zirconia $(\mathrm{Ce}-\mathrm{Zr})$ hybrid nanoparticles are prepared by a bottom-up chemical process namely precipitation method. The synthesized nanoparticles are then subjected to various advanced characterization and stability analysis techniques to determine its size, shape and dispersion stability.

\section{Experimental Work}

\subsection{Materials}

The nano-sized Ceria $(\mathrm{CeO} 2)$ and Ceria-Zirconia $(\mathrm{Ce}-\mathrm{Zr})$ hybrid nanoparticles are prepared chemically. The precursors used for the preparation of former include Cerium (III) Nitrate Hexahydrate, Iso-propanol and water. Further, the Ce-Zr hybrid nanoparticles are prepared from Cerium (III) Nitrate Hexahydrate, Zirconium Oxy Chloride and Water.

\subsection{Synthesis Procedure}

The $0.1 \mathrm{M}$ aqueous solution of precursors, were mixed thoroughly in the desired ratio. The prepared solution was stirred for 30 minutes at $60^{\circ} \mathrm{C}$, by using a magnetic stirrer with heating arrangement. The temperature and the $\mathrm{pH}$ of the solution were checked repeatedly using a $\mathrm{pH}$ meter. Aqueous ammonia was added drop wise to vigorously stirring solution, and the $\mathrm{pH}$ was maintained above the value of 10 to keep the solution basic so that the particles formed will be of nanometre size. On the addition of aqueous ammonia, solution turned pale yellow and mixture was stirred for about 2 hours. The precipitates were collected by filtration and washed repeatedly with distilled water to remove excess ammonia .The slurry was then dried for 4 hours at $500^{\circ} \mathrm{C}$ in a muffle furnace. A porous yellow powder was obtained which was crushed to get fine powder and calcinated at $500^{\circ} \mathrm{C}$ for 1 hour to obtain nano-sized Ceria $(\mathrm{CeO} 2)$ particles. Similar procedure was followed for the synthesis of Ceria-Zirconia (Ce-Zr) hybrid nanoparticles; except that Zirconium Oxy Chloride was used in place of Isopropanol as precursor and the solution turns dark red on addition of ammonium hydroxide solution. A deep yellow powder was obtained in case of Ceria-Zirconia $(\mathrm{Ce}-\mathrm{Zr})$ hybrid nanoparticles.

\subsection{Characterization of Nanoparticles}

The surface morphology and stability analysis of the synthesized nanoparticles were carried out using advanced characterization techniques. HR-TEM using the Jeol/JEM 2100 model with resolution details; Point: $0.23 \mathrm{~nm}$ and Lattice: $0.14 \mathrm{~nm}$ was used to determine the exact size and morphology of the nanoparticles. FT-IR analysis to obtain the absorption spectra was conducted using the Model: Thermo Nicolet, Avatar 370, having a spectral range of 4000-400 cm-1 and maximum resolution of $4 \mathrm{~cm}-1$. XRD plot for interpretation of crystal lattice structure, atomic spacing and to confirm the formation of the desired nanoparticles was performed using the Model: Bruker AXS D8 Advance, with Copper as the X-ray source which produce rays of wavelength: 1.5406 $\mathrm{A}^{\circ}$. The Zeta potential value which determines the dispersion stability of the nanoparticles was found out using the Zetasizer Nano ZS model. Hexane is used as the base fluid to perform the testing to obtain the ZP value in $\mathrm{mV}$ of the different type of nanoparticles. 


\section{Results and Discussion}

\subsection{Surface Morphology and Stability Analysis}

The various advanced characterization techniques viz. HR-TEM, FT-IR, XRD and ZP analyses were carried out on the synthesized Ceria $(\mathrm{CeO} 2)$ and Ceria-Zirconia $(\mathrm{Ce}-\mathrm{Zr})$ hybrid nanoparticles.

\section{A. High Resolution Transmission Electron Microscopy (HR-TEM)}

HR-TEM is an advanced characterization equipment used to determine the surface topography and morphology of materials and particles. To be specific, this ideal equipment is one of the recent developments in the field of microscopic studies for high resolution requirements in researches. Fig. 1 and Fig. 2 show the HR-TEM images of nano-sized Ceria $(\mathrm{CeO} 2)$ and Ceria-Zirconia $(\mathrm{Ce}-\mathrm{Zr})$ hybrid nanoparticles. The results proved that the size of the both type of nanoparticles were much less than $50 \mathrm{~nm}$ and majority of particles seem to have size of nearly $10 \mathrm{~nm}$.
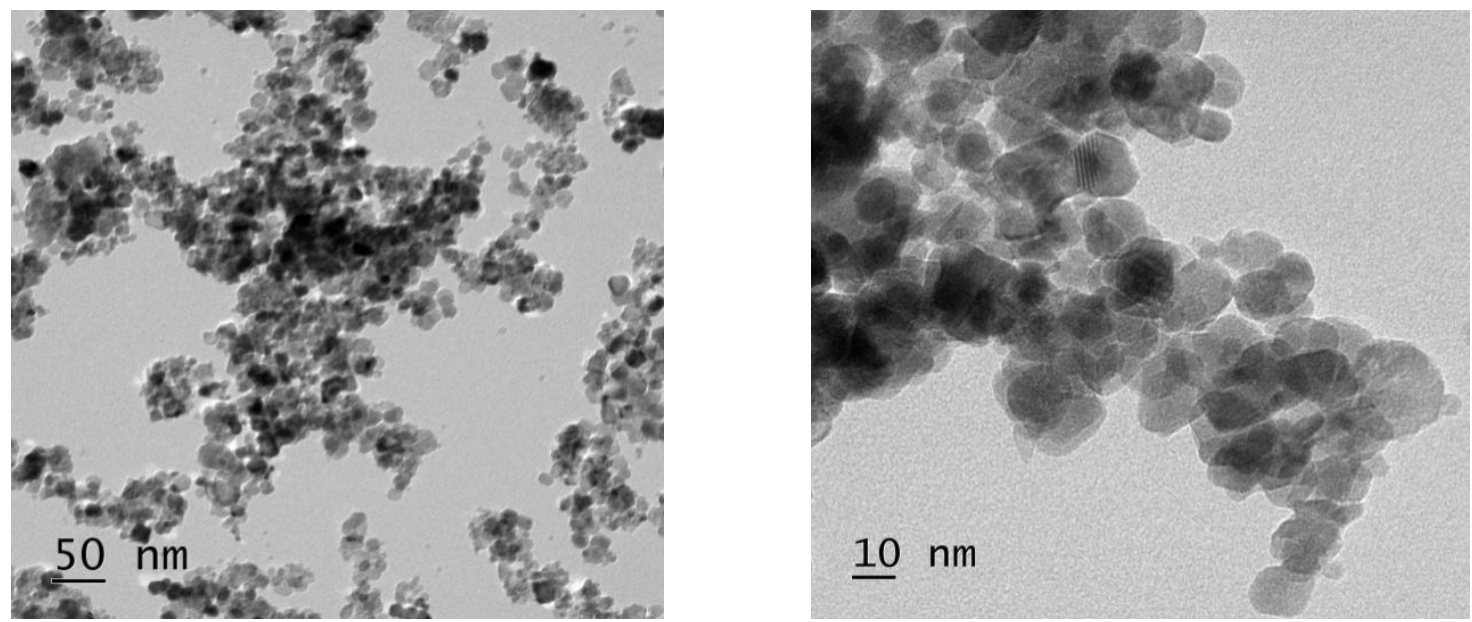

Fig. 1. HR-TEM images of $\mathrm{CeO}_{2}$ nanoparticles
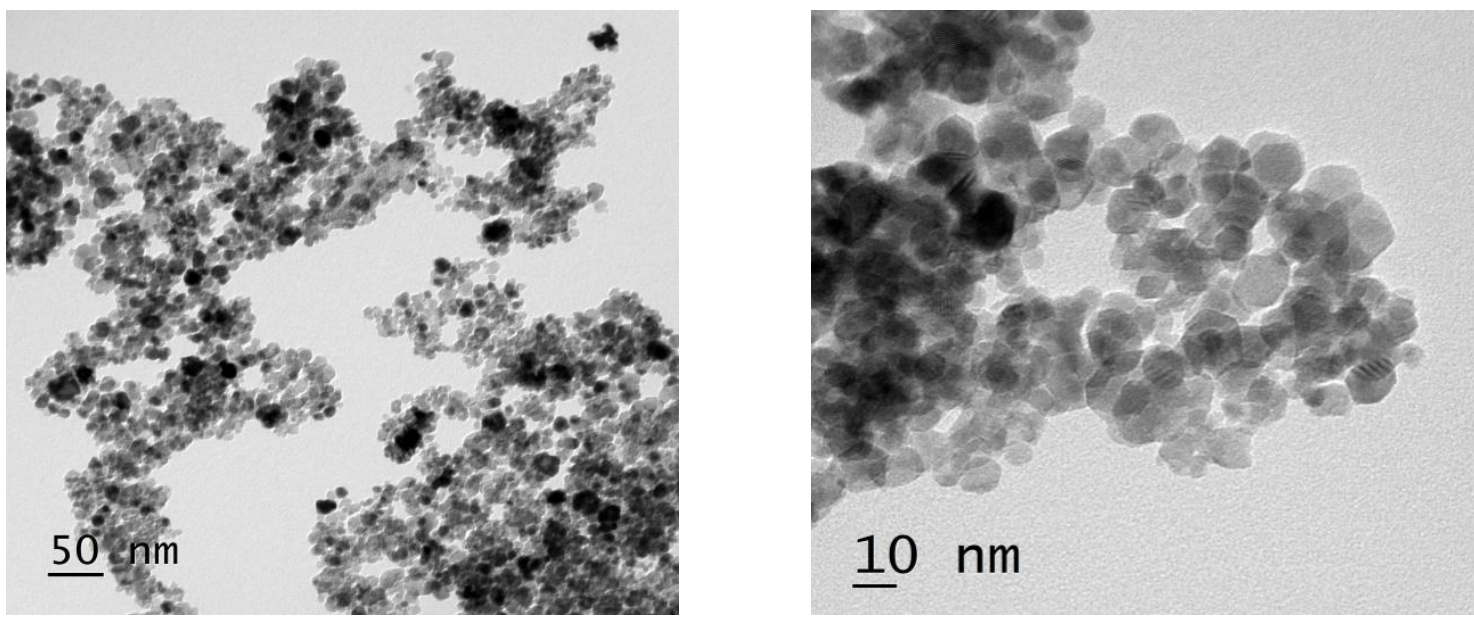

Fig. 2. HR-TEM images of Ce-Zr hybrid nanoparticles

The nearly spherical morphology and polycrystalline nature of both the nano-sized Ceria (CeO2) and CeriaZirconia (Ce-Zr) hybrid nanoparticles can be clearly identified from Fig. 3 obtained using HRTEM. These characteristics are found to be critical in the use of these nanoparticles as lubricant additives. 

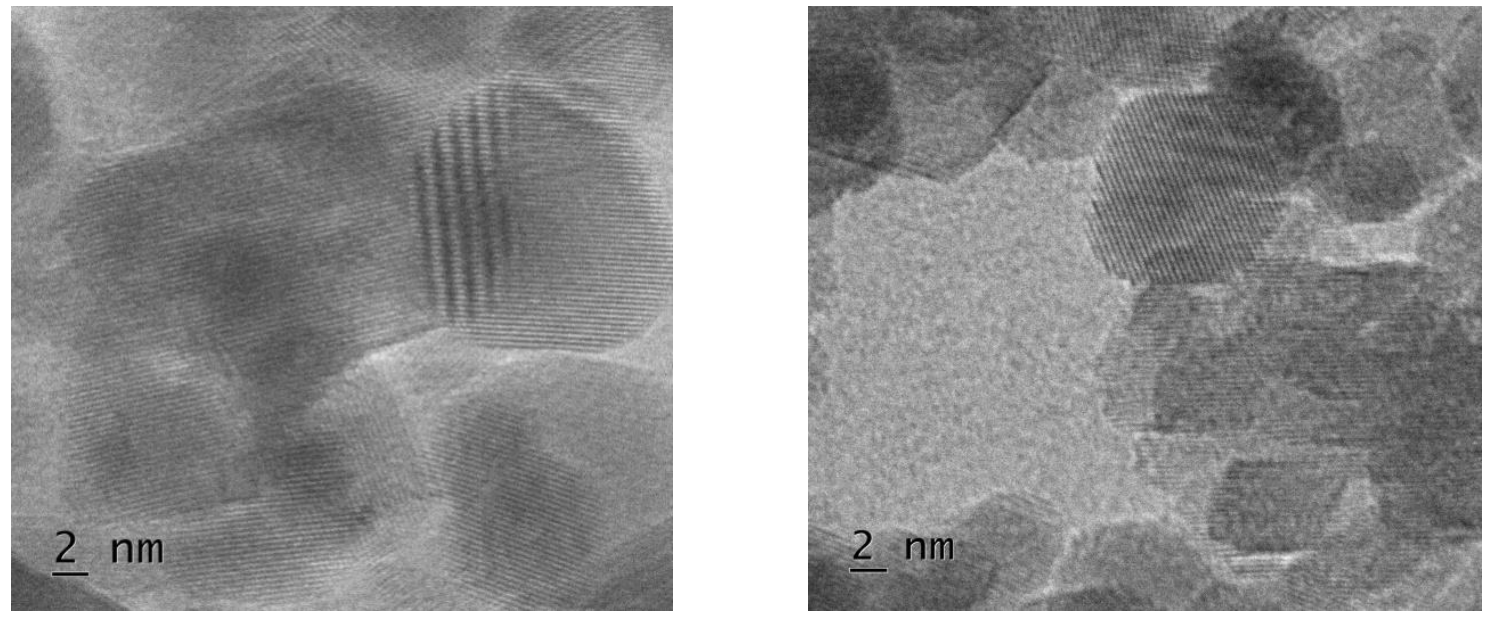

Fig. 3. HR-TEM images showing crystalline nature of both nanoparticles

The Scanning Electron Microscopy (SEM) images of agglomerated nanometer sized Ceria (CeO2) and CeriaZirconia $(\mathrm{Ce}-\mathrm{Zr}$ ) hybrid nanoparticles due to exposure to moisture and improper calcination resulting in larger particles is as shown in Fig. 4.
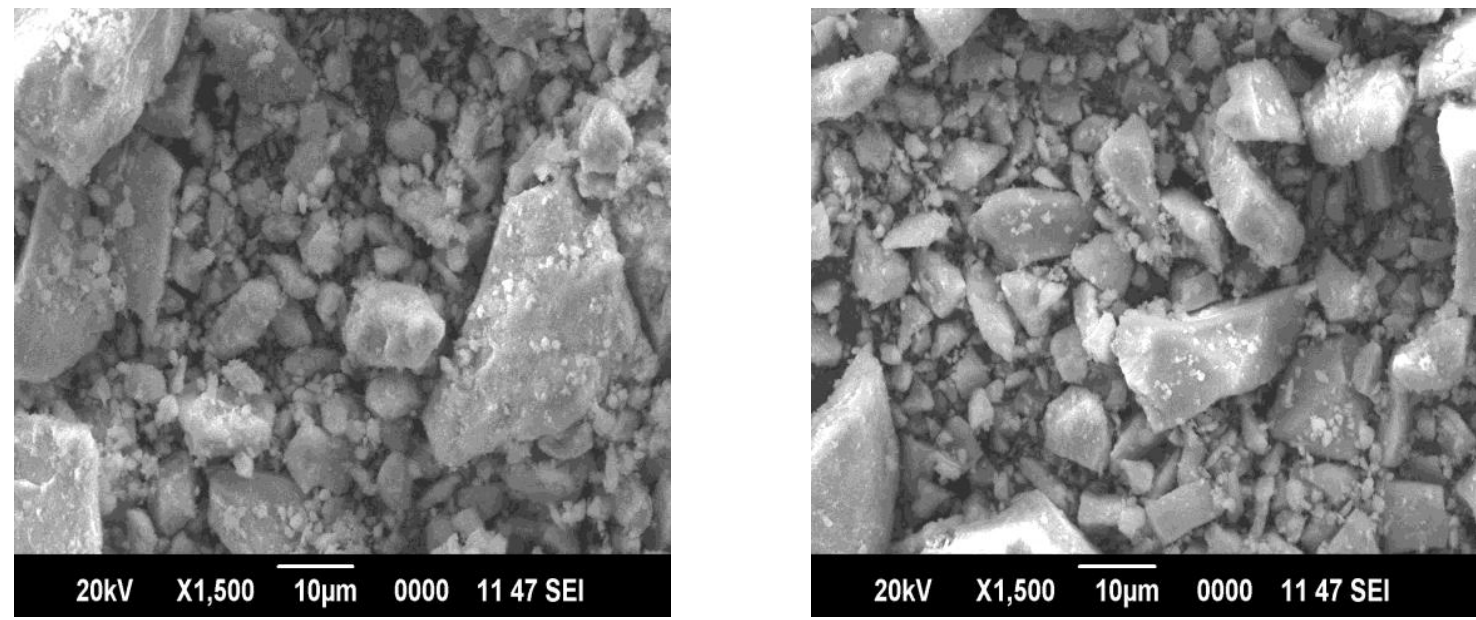

Fig. 4. SEM images of agglomerated $\mathrm{CeO}_{2}$ and $\mathrm{Ce}-\mathrm{Zr}$ nanoparticles to form larger sized particles

The particle size variation is too large which can be undesirable. Further, it can be seen that the shape of the particles get changed to irregular than spherical and measures should be taken to prevent the same to achieve the required spherical nanometer sized particles.

B. Fourier Transform Infrared Spectroscopy (FT-IR)

FT-IR analysis is used to derive the qualitative and quantitative information about the samples being tested. The purity of the synthesized nanoparticles can be verified and the presence of any impurities can be identified by this technique. The FTIR spectra of $\mathrm{CeO} 2$ and surfactant modified $\mathrm{CeO} 2$ nanoparticles is as shown in Fig. 5. The absorption peaks recorded between the wavenumbers of 4000 to $400 \mathrm{~cm}-1$ has a number of significant peaks which are considered. 

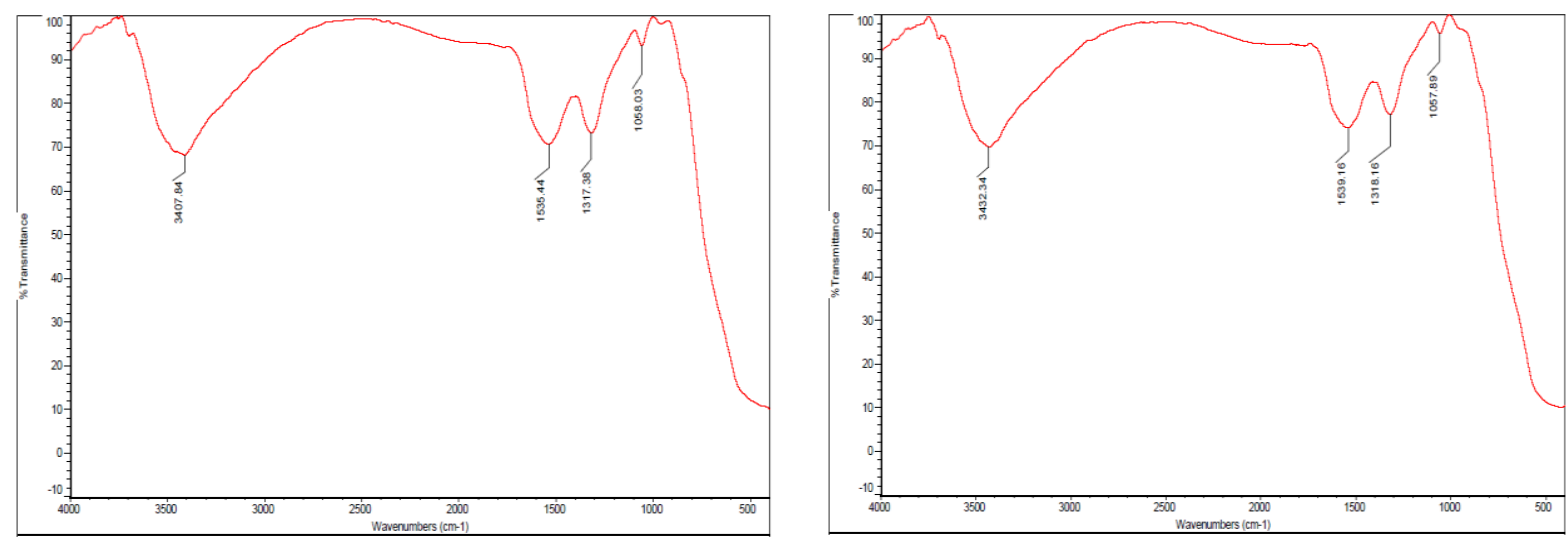

Fig. 5. FT-IR spectra of $\mathrm{CeO}_{2}$ and surfactant modified $\mathrm{CeO}_{2}$ nanoparticles

The absorption peak from $2700-3500 \mathrm{~cm}-1$ is due to the vibrations caused by $\mathrm{O}-\mathrm{H}$ stretching of $\mathrm{H} 2 \mathrm{O}$ in the sample. The water molecules absorbed physically resulted in the absorption bands at 1525.44 and $1317.38 \mathrm{~cm}^{-1}$. Moreover, the typical peak for Ce-O stretching vibrations is featured by the absorption bands at 840 and $540 \mathrm{~cm}^{-}$ ${ }^{1}[13]$.

The FT-IR spectra of Ce-Zr hybrid and surfactant modified hybrid nanoparticles are shown in Fig. 6. Again, the peak at 3425.29 and $1623.73 \mathrm{~cm}^{-1}$ in the spectra is due to the $\mathrm{O}-\mathrm{H}$ stretching vibrations and externally absorbed water molecules. Further, the absorption peaks at 540 and $400 \mathrm{~cm}-1$ is due to the $\mathrm{Ce}-\mathrm{O}$ and $\mathrm{Zr}-\mathrm{O}$ stretching vibrations respectively [13-15]. These adsorption spectra have proved the formation of the desired metal oxide particles to some extend.
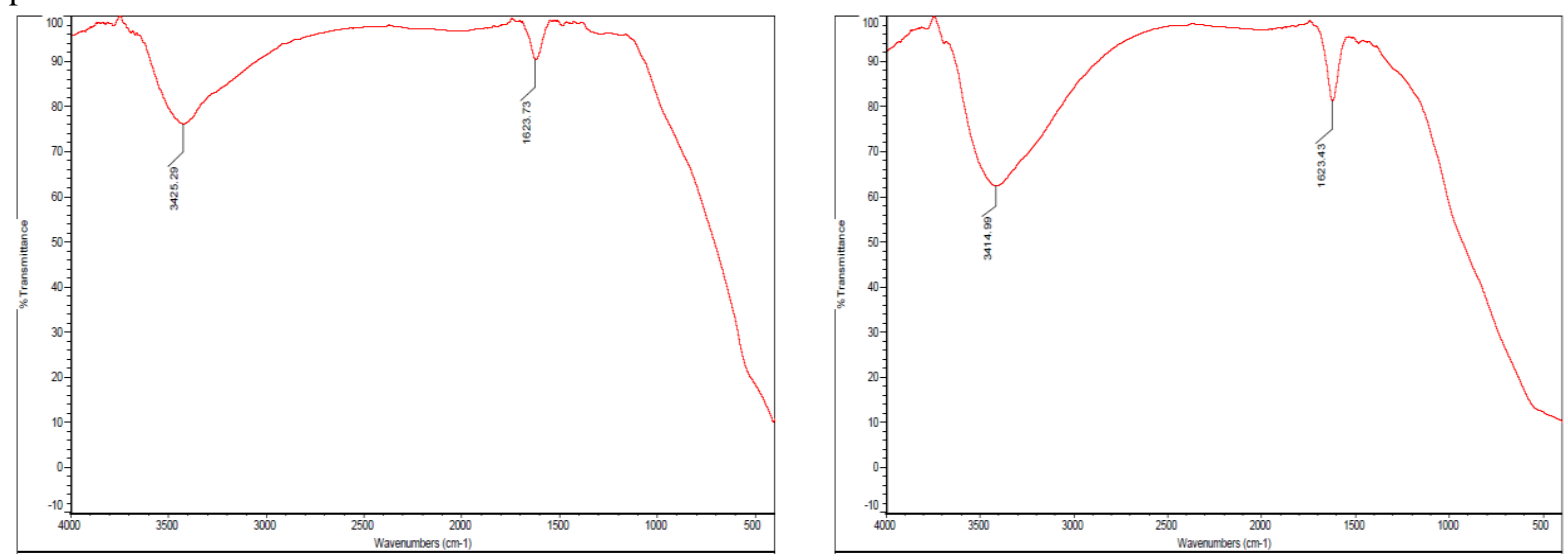

Fig. 6. FT-IR spectra of Ce-Zr hybrid and surfactant modified $\mathrm{Ce}-\mathrm{Zr}$ hybrid nanoparticles

The FT-IR spectra of surfactant modified Ceria $(\mathrm{CeO} 2)$ and Ceria-Zirconia $(\mathrm{Ce}-\mathrm{Zr})$ hybrid nanoparticles does not show much variations form the spectra's related to their respective bare particles, which can be considered as the evidence for absence of chemical reaction of surfactants with- the nanoparticles.

\section{X-Ray Diffraction (XRD)}

Fig. 7 and shows the XRD patterns of Ceria $(\mathrm{CeO} 2)$ and Ceria-Zirconia (Ce-Zr) hybrid nanoparticles respectively. Sharp peaks are obtained for the former and broader peaks for the latter with a shift in all diffraction peaks towards higher $2 \theta$ angles. 

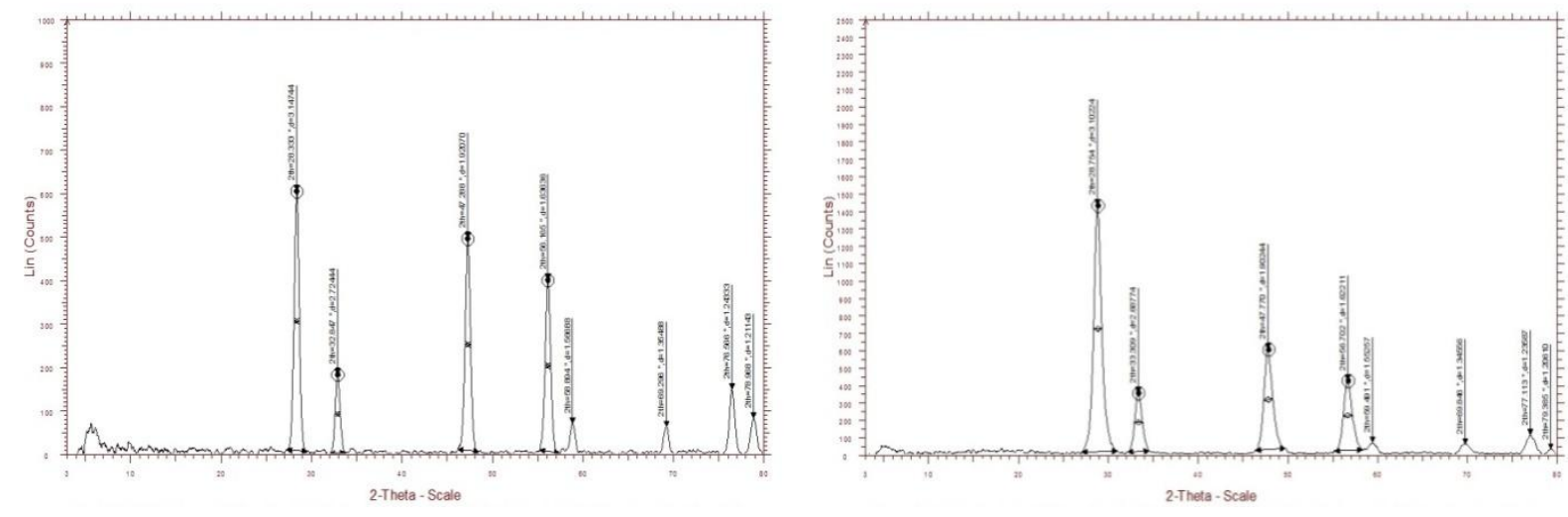

Fig. 7. XRD pattern of $\mathrm{CeO}_{2}$ and $\mathrm{Ce}-\mathrm{Zr}$ hybrid nanoparticles

The peaks obtained for Ceria $(\mathrm{CeO} 2)$ and Ceria-Zirconia $(\mathrm{Ce}-\mathrm{Zr})$ hybrid nanoparticles corresponding to the planes (111), (200), (220), (311) which are similar to that of a face centered cubic fluorite structure. The average particle size of both types of nanoparticles can be obtained using Scherrer's equation:

$$
\text { Dhkl }=0.9 \lambda / \beta \cos \theta
$$

Where Dhkl is the average particle size, $\lambda$ the wavelength of radiation $(0.15406 \mathrm{~nm}), \beta$ the full width at half maximum (FWHM) and $\theta$ the Bragg's angle of the peak. The average particle size and FWHM values of different planes of both type of nanoparticles is summarized in Table 1 and Table 2.

Table 1 Crystalline size and FWHM of $\mathrm{CeO}_{2}$ nanoparticles

\begin{tabular}{|c|c|c|}
\hline Plane & FWHM & Average Particle Size (nm) \\
\hline 111 & $0.614^{\circ}$ & 13.352 \\
\hline 200 & $0.595^{\circ}$ & 13.939 \\
\hline 220 & $0.650^{\circ}$ & 13.870 \\
\hline 311 & $0.637^{\circ}$ & 13.636 \\
\hline
\end{tabular}

Table 2 Crystalline size and FWHM of Ce-Zr hybrid nanoparticles

\begin{tabular}{|c|c|c|}
\hline Plane & FWHM & Average Particle Size (nm) \\
\hline 111 & $0.921^{\circ}$ & 8.9128 \\
\hline 200 & $0.892^{\circ}$ & 9.3073 \\
\hline 220 & $1.048^{\circ}$ & 8.3006 \\
\hline 311 & $1.022^{\circ}$ & 8.8414 \\
\hline
\end{tabular}

Further, comparing the average particle size of both type of particles from HR-TEM images and XRD obtained values we can see that both are in agreement which proves that the results are true. Moreover, the broadening of peaks and their shift towards higher angles of $\mathrm{Ce}-\mathrm{Zr}$ hybrid nanoparticles compared to nanosized $\mathrm{Ceria}(\mathrm{CeO} 2)$ can be due to the reduction in size of the particles.

\section{Zeta Potential Analysis (ZP)}

Zeta potential is the potential difference between the dispersion medium and the stationary layer of fluid attached to the dispersed particle. The magnitude of the zeta potential is a measure of stability of the nanolubricants. The ZP value of nanosized Ceria (CeO2) and Ceria-Zirconia $(\mathrm{Ce}-\mathrm{Zr})$ hybrid nanoparticles dispersed separately in a standard liquid medium of hexane at $30^{\circ} \mathrm{C}$ is as shown in Table 3 . It can be seen that the surfactant modified Ceria-Zirconia (Ce-Zr) hybrid nanoparticles have the highest ZP value of $40.5 \mathrm{mV}$; hence it is the most dispersion stable among all other nanoparticles considered. 
Table 3 ZP values of various nanoparticles considered

\begin{tabular}{|c|l|c|}
\hline Sample No. & \multicolumn{1}{|c|}{ Description } & Zeta Potential (mV) \\
\hline 1 & Hexane $+\mathrm{CeO}_{2}$ & 28.92 \\
\hline 2 & Hexane $+\mathrm{Ce}-\mathrm{Zr}$ hybrid & 31.70 \\
\hline 3 & Hexane + Surfactant modified $\mathrm{CeO}_{2}$ & 38.58 \\
\hline 4 & Hexane + Surfactant modified $\mathrm{Ce}-\mathrm{Zr}$ & 40.50 \\
\hline
\end{tabular}

\section{Conclusions}

The following conclusions are derived on the basis of the characterization process carried out on the nanosized Ceria $\left(\mathrm{CeO}_{2}\right)$ and Ceria-Zirconia $(\mathrm{Ce}-\mathrm{Zr})$ hybrid nanoparticles.

- HR-TEM results proved that both type of nanoparticles have spherical morphology with the majority of particle size ranging from $10 \mathrm{~nm}$ to $20 \mathrm{~nm}$. Further, the crystalline nature of $\mathrm{CeO} 2$ nanoparticles is not lost after doping with Zr. SEM images of the agglomerates nanoparticles proved the need for securing the synthesized particle from atmospheric interactions.

- $\quad$ FT-IR results confirmed the formation of the required metal oxide nanoparticles by PM. Moreover, the absence of chemical interaction of the added surfactant with the nanoparticles can be clearly noted.

- XRD analysis gave an insight into the average particle size of the synthesized nanoparticles, which are compared with the HR-TEM results for confirmation. The reduction in particle size of the $\mathrm{Zr}$ doped nanoparticles can be seen from the data derived of the XRD patterns.

- Finally, the dispersion stability of the surfactant modified nanoparticles over the bare nanoparticles is confirmed from the ZP analysis data.

\section{Acknowledgement}

First of all, we would like to thank God Almighty for giving us enough wisdom and knowledge for successful completion of this research paper. Secondly, we convey our sincere gratitude to Kerala State Council for Science Technology and Environment (KSCSTE), Triruvananthapuram for providing the financial assistance for this initiative.

\section{References}

[1]. Hsu, S. M. (2004). Nano-lubrication: concept and design. Tribology International, 37(7), 537-545.

[2]. Dubey, M. K., Bijwe, J., \& Ramakumar, S. S. V. (2013). PTFE based nano-lubricants. Wear, 306(1), 80-88.

[3]. Koshy, C. P., Rajendrakumar, P. K., \& Thottackkad, M. V. (2015). Evaluation of the tribological and thermo-physical properties of coconut oil added with MoS2 nanoparticles at elevated temperatures. Wear, 330, 288-308.

[4]. Wu, Y. Y., Tsui, W. C., \& Liu, T. C. (2007). Experimental analysis of tribological properties of lubricating oils with nanoparticle additives. Wear, 262(7), 819-825.

[5]. Koshy, C. P., Rajendrakumar, P. K., \& Thottackkad, M. V. (2014). Experimental Evaluation of the Tribological Properties of $\mathrm{CuO}$ NanoLubricants at Elevated Temperatures. In Proceedings of International Conference on Advances in Tribology and Engineering Systems (pp. 391-402). Springer India.

[6]. Koshy, C. P., Rajendrakumar, P. K., \& Thottackkad, M. V. (2015). Analysis of Tribological and Thermo-Physical Properties of SurfactantModified Vegetable Oil-Based CuO Nano-Lubricants at Elevated Temperatures-An Experimental Study. Tribology Online, 10(5), 344-353.

[7]. Chen, H. I., \& Chang, H. Y. (2004). Homogeneous precipitation of cerium dioxide nanoparticles in alcohol/water mixed solvents. Colloids and Surfaces A: Physicochemical and Engineering Aspects, 242(1), 61-69.

[8]. Verdon, E., Devalette, M., \& Demazeau, G. (1995). Solvothermal synthesis of cerium dioxide microcrystallites: effect of the solvent. Materials letters, 25(3), 127-131.

[9]. Yadav, T. P., \& Srivastava, O. N. (2012). Synthesis of nanocrystalline cerium oxide by high energy ball milling. Ceramics International, 38(7), 5783-5789. 
[10]. Zhou, F., Zhao, X., Xu, H., \& Yuan, C. (2007). CeO2 spherical crystallites: synthesis, formation mechanism, size control, and electrochemical property study. The Journal of Physical Chemistry C, 111(4), 1651-1657.

[11]. Zhou, W., \& Wang, Z. L. (2007). Scanning microscopy for nanotechnology: techniques and applications. Springer science \& business media.

[12]. Spikes, H. (2015). Friction Modifier Additives. Tribology Letters, 60(1), 1-26.

[13]. Babitha, K. K., Sreedevi, A., Priyanka, K. P., Sabu, B., \& Varghese, T. (2015). Structural characterization and optical studies of $\mathrm{CeO} 2$ nanoparticles synthesized by chemical precipitation. Indian Journal of Pure \& Applied Physics (IJPAP), 53(9), 596-603.

[14]. Zhang, H., Liu, Y., Zhu, K., Siu, G., Xiong, Y., \& Xiong, C. (1999). Infrared spectra of nanometre granular zirconia. Journal of Physics: Condensed Matter, 11(8), 2035.

[15]. Sinha, N., Ray, G., Bhandari, S., Godara, S., \& Kumar, B. (2014). Synthesis and enhanced properties of cerium doped $\mathrm{ZnO}$ nanorods. Ceramics International, 40(8), 12337-12342. 